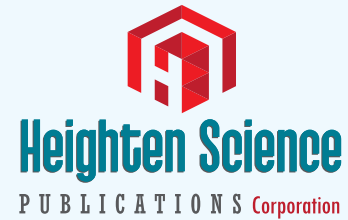

ISSN

2640-284X
*Address for Correspondence: Dr. Sajjad Ahsan, Department of Endocrinology, Indira Gandhi Institute of Medical Sciences, Patna Bihar, India, Email: sajjad.ahsan@rediffmail.com

Submitted: 29 August 2018

Approved: 12 September 2018

Published: 13 September 2018

Copyright: @ 2018 Ranjan P, et al. This is an open access article distributed under the Creative Commons Attribution License, which permits unrestricted use, distribution, and reproduction in any medium, provided the original work is properly cited

Keywords: Hydroxychloroquine; Teneligliptin Insulin; Type 2 Diabetes; HbA1c

\title{
Comparison of Efficacy and Safety of Hydroxychloroquine and Teneligliptin in Type 2 Diabetes Patients who are Inadequately Controlled with Glimepiride, Metformin and Insulin therapy: A Randomized Controlled Trial with Parallel Group Design
}

\author{
Prakash Ranjan', Sajjad Ahsan ${ }^{2 \star}$, Rabi Bhushan ${ }^{1}$, Bipin Kumar ${ }^{3}$, \\ Tushar ${ }^{4}$, Anup Kumar Gupta ${ }^{1}$, Anand Kumar Verma ${ }^{1}$ and \\ Mukesh Jain ${ }^{1}$ \\ 'The National Institute of Health and Family Welfare, New Delhi, India \\ 2Indira Gandhi Institute of Medical Sciences, Patna, Bihar, India \\ ${ }^{3}$ Patna Medical College and Hospital, Patna, Bihar, India \\ ${ }^{4}$ Darbhanga Medical College and Hospital, Darbhanga, Bihar, India
}

\section{Abstract}

Aim: The aim of the present study is to assess the efficacy and safety of Hydroxychloroquine in comparison with Teneligliptin in type 2 diabetes patients whose blood glucose levels were inadequately controlled with metformin, Glimepiride and insulin therapy.

Methods: This was a randomized, prospective, parallel-group, experimental trial done in 300 Type 2 Diabetes patients who were uncontrolled (HbA1c=7.5-10\%) with metformin, Glimepiride and insulin therapy. Patients were randomly divided into two groups one received Teneligliptin $20 \mathrm{mg}(\mathrm{n}=152)$ and other received Hydroxychloroquine $400 \mathrm{mg}(\mathrm{n}=148)$ while continuing insulin therapy with other $2 \mathrm{OHA}$. Insulin doses were adjusted to maintain normal blood glucose levels.

Result: The adjusted mean change from baseline to endpoint in $\mathrm{HbA} 1 \mathrm{c}$ was $-1.2 \pm 0.5 \%$ in patient group receiving Hydroxychloroquine and $-0.9 \pm 0.5 \%$ in patients group receiving Teneligliptin, respectively, with a significant between-treatment difference $(\mathrm{p}<0.001)$. The incidence of adverse events was similar in the Hydroxychloroquine (72\%) and Teneligliptin (77\%) groups. However, hypoglycaemic events were less common $(p<0.001)$ and less severe $(p<0.05)$ in patients receiving Hydroxychloroquine than in those receiving Teneligliptin.

Conclusion: Hydroxychloroquine decreases $\mathrm{HbA} 1 \mathrm{c}$ in patients whose type 2 diabetes is poorly controlled with high doses of insulin as compare to Teneligliptin. Addition of hydroxychloroquine to insulin therapy is also associated with reduced incidence of confirmed and severe hypoglycaemia.

\section{Introduction}

Diabetes is progressive in nature and the substantial evidence supporting the beneficial effects of insulin regimens, as it not only maintains glycemic control as well as reduce morbidity and mortality rates associated with diabetes and its related complications [1,2]. Insulin therapy strongly ameliorates hyperglycemia but has 
adverse effects such as hypoglycemia [3] and weight gain, which might increase the incidence of cardiovascular events. These adverse events can be minimized by the initial use of insulin in combination with oral antidiabetic agents [4]. The advantages of concomitant therapy are not only improvement of glycemic control but also a reduction in the number of insulin injections and titration of the insulin dose.

Teneligliptin has unique J shaped or anchor locked domain structure because of which it has a potent inhibition of DPP 4 enzyme. Teneligliptin significantly controls glycemic parameters with safety. Teneligliptin have a structure which is unique and binds to S1, S2, and S2 extensive sub site of DPP-4 enzyme. It is recommended Once-aday administration [5]. Moreover multiple trails [6-9] on DPP-4 inhibitors have shown better glycemic control in type 2 diabetes mellitus patients and these patients had minimal risk of hypoglycemia and weight gain.

Hydroxychloroquine inhibits insulin degradation and improve insulin sensitivity. A few randomized controlled trials showed that HCQ lowers HbA1c and LDL cholesterol levels in patients with type 2 diabetes [10]. Furthermore, in a prospective, randomized, placebo, double-blind 6-month trial the addition of Hydroxychloroquine to either insulin or glibenclamide in the treatment of refractory noninsulin-dependent diabetes for 6 months resulted in a significant decrease in HbA1C by $3.3 \%$ compared with placebo as well as a reduction in insulin dose by $30 \%$ in the insulin-treated group [11]. In a recent RTCT trial conducted by Baidya et al. [12], confirms that in type 2 diabetes patients who are poorly controlled, addition of hydroxychloroquine (HCQ) to insulin along with Glimepiride and Metformin, there was significant overall mean change in total daily insulin dose at study end by almost $28 \%$ with a HbA1c reduction of $-1.3 \%$ from baseline within 6 months. When Hydroxychloroquine was evaluated against Teneligliptin in patient who were uncontrolled on Metformin $2000 \mathrm{mg}$ and Glimepiride $2 \mathrm{mg}$, a further HbA1c reduction of $-1.8 \%$ happened with Hydroxychloroquine than compared to Teneligliptin with which it was $-1.3 \%$ [13]. Even in few trials $[14,15]$ Hydroxychloroquine exhibit a potent efficacy even comparable to other DPP4 inhibitors like Vildagliptin and Sitagliptin.

Themainpurpose of the trialtocomparetheefficacyand safetyofHydroxychloroquine and Teneligliptin in Type 2 Diabetes Patients Who Are Inadequately Controlled with Glimepiride, Metformin and Insulin therapy. This is particularly important because as diabetes progresses its management becomes increasingly difficult. Patients often have multiple comorbidities requiring pharmacological treatment. Due to contraindications this can limit therapeutic options and may also lead to suboptimal patient compliance. Hypoglycaemia may become a limiting factor in the treatment of patients with advanced disease [16]. Moreover, insulin-treated patients with type 2 diabetes are often profoundly insulin-resistant and require high doses, which can exacerbate weight gain.

\section{Subjects and Methods}

\section{Study design and study population}

This was a randomized, prospective, parallel-group, experimental trial conducted at an outpatient facility in 300 Type 2 Diabetes patients who were uncontrolled (HbA1c=7.5-10\%) with metformin, sulfonylurea (Glimepiride) and insulin therapy. Patients were randomly divided into two groups one received Teneligliptin $20 \mathrm{mg}$ $(n=152)$ and other received Hydroxychloroquine $400 \mathrm{mg}(\mathrm{n}=148)$ while continuing insulin therapy with other two OHA. To be eligible, patients had to have received injectable insulin for at least 3 months along with specified dose of metformin and glimepiride, at a dose of at least $30 \mathrm{U} /$ day for a minimum of 4 weeks prior to enrolment. Male and female were eligible upon fulfilment of the following conditions: aged 1880 years; HbA1c=7.5-10\%; fasting plasma glucose $(\mathrm{FPG})<200 \mathrm{mg} / \mathrm{dl}$, Post Prandial Plasma glucose $(\mathrm{PPG})<350 \mathrm{mg} / \mathrm{dl}$ and BMI 22-45 kg/m2. Patients were excluded 
if they had any severe complications of diabetes. Patients with recent $(<1$ year) CV events i.e. myocardial infarction/ACS, stroke or has undergone coronary artery bypass surgery, percutaneous transluminal coronary angioplasty or transient ischemic attack or history of congestive heart failure or unstable angina, severe renal and liver dysfunction, severe infections. Patient with known history of diabetic ketoacidosis, Glucose-6-phophodehydrogenase deficiency, existing diabetic retinopathy of any grade including diabetic retinopathy requiring laser therapy, evidence of an imminent need for retinal laser therapy, uncorrected visual acuity $<20 / 100$, abnormal visual fields, difficulty to examine optic disc or evidence of retinal pigment epithelial abnormalities and patients with history or risk of macular edema.

Of the 356 subjects screened for this study, 320 subjects were randomized to either the Hydroxychloroquine or teneligliptin group. A total of 36 subjects was screened but not randomized. Of these 36 subjects, 29 were excluded for unmet eligibility criteria ( 24 for inclusion and 5 for exclusion criteria). The other 7 subjects were excluded for the following reasons: 2 subject's was recommended to be hospitalized for blood glucose control by the investigator, another 5 was not able to be contacted and was withdrawn from the study due to problems related to patient's diary. Among 320 randomised subjects 6 patients in Hydroxychloroquine group and 4 patients on teneligliptin group excluded because of protocol violation, 5 patients in hydroxychloroquine group and 3 patients in teneligliptin group has lost in follow up. In both the group one patient each had died due to some other reason.

\section{Ethical consideration}

This study was conducted in accordance with the good clinical practice guidelines and with the Helsinki Declaration principles. Individual ethical committee approval was obtained prior to the trial. Also prior to conduction of the study related procedure/ investigation, a voluntary written informed consent was taken from the patient / legally acceptable representative.

\section{Study assessments}

The primary efficacy parameter was the change from baseline to Week 24 or endpoint in HbA1c for Week 24. Secondary efficacy parameters included FPG, mean daily insulin dose, mean daily number of insulin injections, fasting lipid parameters (Total cholesterol, LDL-C, HDL-C, Triglyceride) and body weight. HbA1c, FPG, PPG, insulin dose regimen and body weight were recorded at each study visit. Lipid profiles were assessed at weeks $-4,0,4,12$ and 24 . All assessments were made by accredited lab. Although insulin dose adjustments were allowed during the study, patients were asked to use the same type of insulin throughout, and if possible to maintain their daily insulin regimen. Investigators were allowed to decrease a patient's insulin dose according to their clinical judgement in the event of severe or repeated hypoglycaemic episodes. Upward dose adjustments were allowed as clinically indicated, but were recommended to remain within $25 \%$ of the baseline insulin dose.

\section{Safety}

All adverse events were recorded and assessed by the investigator as to severity and possible relationship to study medication. Vital signs were recorded at each study visit; safety laboratory assessments were made at weeks -4, 0, 4, 12 and 24; ECGs were performed at weeks $-4,0,4,12$ and Patients were asked to use glucose monitoring devices and instructed on their use. It was ensure that all patients under this study uses the same type of device with a view to performing routine self-monitored blood glucose (SMBG) measurements as instructed by the investigator. They were asked to record the measured values at any time hypoglycaemia was suspected and also prior to breakfast at least three times per day. Confirmed hypoglycaemia was defined as symptoms suggestive of low blood glucose confirmed by an SMBG measurement $<70$ 
$\mathrm{mg} / \mathrm{dl}$ plasma glucose equivalent. Severe hypoglycaemia was defined as any episode requiring the assistance of another party (with plasma glucose value $<70 \mathrm{mg} / \mathrm{dl}$, unless the severity of the event precluded glucose determination).

\section{Statistical analysis}

All statistical analyses were performed using statistical software (Graph Pad Prism, version 6). Data are presented as mean \pm SD values. Differences between times points were assessed using the Wilcoxon signed rank test. A value of $\mathrm{P}<0.05$ was considered significant.

\section{Result}

The demographics and clinical characteristics of the randomized subjects are summarized in table 1. For the Hydroxychloroquine group, 53\% of the subjects were male, the mean age was $55 \pm 8 \mathrm{yr}$, and duration of diabetes was $9 \pm 3$ yr. For Teneligliptin group, $53 \%$ of subjects were male, the mean age was $56 \pm 9 \mathrm{yr}$ and duration of diabetes was $10 \pm 2 \mathrm{yr}$. The demographics and baseline characteristics of Hydroxychloroquine group were comparable to Teneligliptin group and there were no significant differences between groups (Figure 1).

\begin{tabular}{|c|c|c|c|}
\hline Characteristic & $\begin{array}{l}\text { HCQ Treatment Group } \\
\qquad N=148\end{array}$ & $\begin{array}{l}\text { Teneligliptin Treatment Group } \\
\qquad N=152\end{array}$ & $P$ value \\
\hline Age (years) & $55 \pm 8$ & $56 \pm 9$ & 0.59 \\
\hline Gender (Male)\% & $79(53 \%)$ & $84(55 \%)$ & 0.49 \\
\hline Body mass index (kg/m2) & $25.7 \pm 2.4$ & $25.3 \pm 2.0$ & 0.77 \\
\hline Disease duration (years) & $9 \pm 3$ & $10 \pm 2 @$ & 0.65 \\
\hline $\mathrm{HbA} 1 \mathrm{c}(\%)$ & $8.3 \pm 0.5$ & $8.2 \pm 0.5$ & 0.54 \\
\hline FBG (mg/dl) & $134.03 \pm 16.44$ & $135.07 \pm 16.51$ & 0.58 \\
\hline PBG (mg/dl) & $239.89 \pm 21.5$ & $234.72 \pm 23.1$ & 0.73 \\
\hline Total cholesterol (mg/dL) & $159.71 \pm 25.43$ & $163.08 \pm 26.94$ & 0.563 \\
\hline LDL-C (mg/dL) & $104.54 \pm 18.57$ & $105.21 \pm 16.61$ & 0.741 \\
\hline HDL-C (mg/dL) & $46.64 \pm 10.48$ & $49.69 \pm 13.34$ & 0.182 \\
\hline Triglyceride (mg/dL) & $132.81 \pm 35.80$ & $131.87 \pm 32.91$ & 0.813 \\
\hline Total daily insulin dosage (IU/day) & $35.8 \pm 16.2$ & $36.9 \pm 17.3$ & 0.046 \\
\hline $\begin{array}{l}\text { Time of insulin injections (times/ } \\
\text { day) }\end{array}$ & $3.2 \pm 1.0$ & $3.3 \pm 1.1$ & 0.16 \\
\hline \multicolumn{4}{|l|}{ Type of insulin } \\
\hline Prandial insulin & $59(40 \%)$ & $61(40 \%)$ & 0.49 \\
\hline Prandial insulin dosage (IU/day) & $41.2 \pm 17.2$ & $39.4 \pm 12.9$ & 0.6 \\
\hline Premixed insulin & $48(32 \%)$ & $49(32 \%)$ & 0.72 \\
\hline Premixed insulin dosage (IU/day) & $35.1 \pm 15.3$ & $38.3 \pm 12.2$ & 0.02 \\
\hline Basal insulin & $41(28 \%)$ & $42(28 \%)$ & 0.92 \\
\hline Basal insulin dosage (IU/day) & $31.2 \pm 13.7$ & $33.2 \pm 14.1$ & 0.3 \\
\hline
\end{tabular}

Figure 1: Study flow diagram.




\section{Change of HbA1c, FBG and PPG}

Table 2 presents the change in HbA1c from baseline to week 24 as well as the difference between the groups. At week 24, in Hydroxychloroquine group the mean HbA1c decreased from $8.3 \pm 0.5 \%$ to $7.1 \pm 0.5 \%$ in teneligliptin group and from 8.2 $\pm 0.5 \%$ to $7.3 \pm 0.5 \%$, respectively. P value in-between two groups were statistically significant. The mean fasting blood glucose level at week 24 decreased by $21.677 \pm 12.63$ $\mathrm{mg} / \mathrm{dL}$ in hydroxychloroquine group and $15.25 \pm 14.31 \mathrm{mg} / \mathrm{dL}$ in teneligliptin group. The mean Post Prandial blood glucose level at week 24 decreased by $57.68 \pm 25.61 \mathrm{mg} /$ $\mathrm{dL}$ in Hydroxychloroquine group and $43.1 \pm 22.38 \mathrm{mg} / \mathrm{dL}$ in Teneligliptin group.

\section{Change in BMI}

There is marginal change of BMI in Hydroxychloroquine group as compare to Teneligliptin group but differences in between the groups are not statistically significant.

\section{Change of lipid parameters}

The change in lipid parameter levels (total cholesterol, LDL-C, HDL-C, non-HDL-C, triglycerides) from baseline to week 24 by group, as well as the differences between groups is shown in table 2 . The changes of total cholesterol, triglyceride, LDL cholesterol, HDL cholesterol and non-HDL cholesterol levels between baseline and week 24 in both Hydroxychloroquine and Teneligliptin groups could not show the statistically significant differences, despite there is marginal decrease in triglyceride, LDL and total cholesterol among hydroxychloroquine group with slight increase in HDL.

\section{Change in Insulin dosage and time of insulin injection}

As compare to Teneligliptin group insulin dosage and time of insulin injection there is significant decrease in Hydroxychloroquine group. There was almost 28\% decrease of insulin dosage in Hydroxychloroquine group where as it was 19\% with Teneligliptin group.

\section{Hypoglycemia}

Figure 2 shows the frequency of confirmed (Figure 2a) and severe (Figure 2b) hypoglycaemia during 24 week treatment. Both rates were significantly lower in the Hydroxychloroquine group (1.91 and 0 events per patient-year, respectively) than in

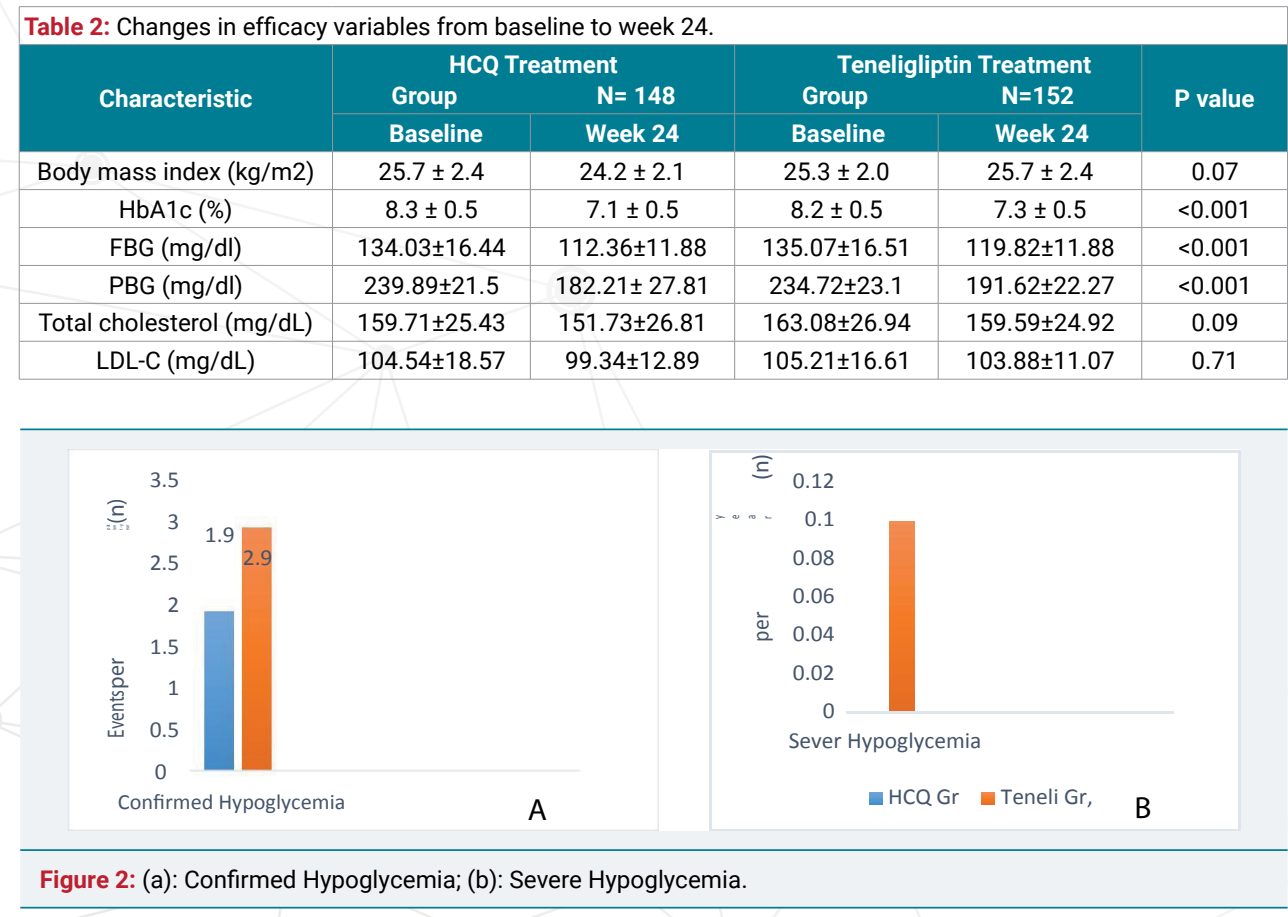


the Teneligliptin group ( 2.98 and 0.10 events per patient-year, respectively; $\mathrm{p}<0.001$ and $p=0.032$ based on $\chi 2$ test of two Poisson rates). Expressed in absolute number of events, 33 patients in the Hydroxychloroquine group reported a total of 113 events, none of which were severe (i.e. requiring assistance of another party). In the Teneligliptin group, 45 patients reported a total of 185 events, six of which were severe.

\section{Adverse events}

When added to insulin therapy, the overall frequency of adverse events in patients receiving Hydroxychloroquine (72\%) was similar to that in patients receiving Teneligliptin (77\%). Other than confirmed hypoglycaemia (detailed above), the most commonly reported specific adverse events in patients receiving Hydroxychloroquine were: abdominal discomfort (18\%), diarrhoea (16\%), asthenia (12\%), hyperhidrosis (16\%), dizziness $(13 \%)$ and headache (9\%). In patients receiving Teneligliptin, the most frequent specific adverse events were abdominal distension (11\%), Dyspepsia $(12 \%)$, tremor (15\%), hyperhidrosis (13\%), dizziness (15\%), asthenia $(13 \%)$ and upper respiratory tract infection (9\%). All other specific adverse events were reported by less than $10 \%$ of patients in either group and there were no remarkable differences between treatment groups in the incidence of any specific adverse event. One patient in the Hydroxychloroquine group died due to malignant lung neoplasm. One patient in the Teneligliptin group died from coronary artery disease. No major changes from baseline to endpoint or between-treatment differences at endpoint were observed for any vital sign and no consistent trends over time were noted. The frequency and nature of ECG changes from baseline to endpoint were comparable in the two treatment groups. Two patients of Teneligliptin group reported mild QT prolongation with Teneligliptin after 6 month of treatment.

\section{Discussion}

Type 2 Diabetes Mellitus is a progressive disease characterized by insulin resistance, impaired insulin secretion, increased glycogen dysplasia and obesity, the incidence of which has been rising consistently worldwide. It is widely recognized to be caused by impaired functions of pancreatic $b$ and a cells [16]. According to a British prospective study, the functions of pancreatic $b$ cells has been decreasing at a rate of $4 \%$ to $5 \%$ each year in diabetic patients, even 10 to 15 years before the diagnosis was made $[17,18]$.

These findings indicate that insulin treated patients with type 2 diabetes may experience improved glycemic control with the addition of Hydroxychloroquine $400 \mathrm{mg}$ as compare to Teneligliptin $20 \mathrm{mg}$. There was $1.2 \%$ reduction in HbA1c with addition of Hydroxychloroquine $400 \mathrm{mg}$ after 24 week of treatment whereas with addition of Teneligliptin $20 \mathrm{mg}$ it was $0.9 \%$. Even there was statistically significant reduction in FBG and PBG in Hydroxychloroquine group as compare to Teneligliptin group $(\mathrm{p}<0.001)$.

Hypoglycaemia is a major limiting barrier to good glycaemic control with insulin [19]. The main finding of this 24-week randomised, controlled trial was that despite an improvement in glycaemic control in patients with type 2 diabetes that was poorly controlled with high doses of insulin, the addition of Hydroxychloroquine was associated with reduced hypoglycaemia, particularly severe hypoglycaemia.

There was almost 28\% decrease of insulin dosage in Hydroxychloroquine group where as it was $19 \%$ with Teneligliptin group. This is accordance with previous trial conducted by Baidya et al. [12], which showed in Indian patients where $28 \%$ insulin dose reduction was observed with Hydroxychloroquine $400 \mathrm{mg}$. In another RCT trial conducted by Quatraro A et al. [11], which showed further 30\% reduction of insulin dose with addition of Hydroxychloroquine $400 \mathrm{mg}$. Change in Insulin dosage and time of insulin injection among Teneligliptin dose was also noticed but compare to Hydroxychloroquine group it was much lesser. This study confirms that prescribers 
should be aware of the gradual onset of action of hydroxychloroquine with maximal effects on HbA1c occurring up to $\geq 3$ months after treatment begins. Insulin dosage may need to be adjusted to avoid or manage hypoglycemia.

These findings conclude that the co-administration of Hydroxychloroquine $400 \mathrm{mg}$ with insulin results in a sustained improvement in glycemic control when administered to patients with type 2 diabetes who are poorly controlled on insulin therapy along with other oral antidiabetic agents. In addition, these findings support early studies suggesting that Hydroxychloroquine improves glycemic control by increasing insulin sensitivity in patients with type 2 diabetes. In other words, irrespective of the history, body habitus, insulin dose and insulin secretory capacity, concomitant therapy with Hydroxychloroquine $400 \mathrm{mg}$ is likely to be effective in patients with poor glycemic control. Stratified analysis based on the insulin regimen suggested that add-on therapy with sitagliptin is most effective when combined Teneligliptin with insulin therapy. Based on the encouraging results of this study, further investigation on a larger scale is warranted in the future to confirm it further.

\section{Conclusion}

Hydroxychloroquine decreases HbA1c in patients whose type 2 diabetes is poorly controlled with high doses of insulin as compare to Teneligliptin. Addition of hydroxychloroquine to insulin therapy is also associated with reduced incidence of confirmed and severe hypoglycaemia.

\section{Acknowledgment}

The authors declare no financial support or relationships that may pose a conflict of interest. Authors acknowledge the immense help received from the scholars whose articles are cited and included in references of this manuscript. The authors are also grateful to authors /editors / publishers of all those articles, journals and books from where the literature for this article has been reviewed and discussed.

\section{References}

1. Nathan DM, Cleary PA, Backlund JY, Genuth SM, Lachin JM, et al. Intensive diabetes treatment and cardiovascular disease in patients with type 1 diabetes. N Engl J Med. 2005; 353: 2643-2653. Ref.: https://goo.gl/tNvjBX

2. Bergenstal RM, Johnson M, Powers MA, Wynne A, Vlajnic A, et al. Adjust to target in type 2 diabetes: comparison of a simple algorithm with carbohydrate counting for adjustment of mealtime insulin glulisine. Diabetes Care. 2008; 31: 1305-1310. Ref.: https://goo.gl/GTm5gb

3. Inzucchi SE, Bergenstal RM, Buse JB, Diamant M, Ferrannini E, et al. Management of hyperglycaemia in type 2 diabetes: a patient-centered approach: position statement of the American Diabetes Association (ADA) and the European Association for the Study of Diabetes (EASD). Diabetologia. 2012; 55: 1577-1596. Ref.: https://goo.gl/tUCDCC

4. Donner T, Munoz M. Update on insulin therapy for type 2 diabetes. J Clin Endocrinol Metab. 2012; 97: 1405-1413. Ref.: https://goo.gl/sTivNH

5. Kishimoto M. Teneligliptin: a DPP-4 inhibitor for the treatment of type 2 diabetes. Diabetes Metab Syndr Obes. 2013; 6: 187-195. Ref.: https://goo.gl/MxuEBg

6. Tsuchimochi W, Ueno H, Yamashita E, Tsubouchi C, Sakoda H, et al. Teneligliptin improves glycemic control with the reduction of postprandial insulin requirement in Japanese diabetic patients". Endocr J. 2015; 62: 13-20. Ref.: https://goo.gl/YPbJth

7. Ghosh S, Trivedi S, Sanyal D, Modi KD, Kharb S. Teneligliptin real-world efficacy assessment of type 2 diabetes mellitus patients in India (TREAT- INDIA Study). Diabetes Metab Syndr Obes. 2016; 9: 347-353. Ref.: https://goo.gl/vJeUqd

8. Scott LJ. Teneligliptin; a review in type 2 diabetes. Clin Drug Investig. 2015; 35: 765-772. Ref.: https://goo.gl/2RcsJh

9. Kim MK, Rhee EJ, Han KA, Woo AC, Lee MK, et al. Efficacy and safety of teneligliptin: a dipeptidyl peptidase 4 inhibitor, combined with metformin in Korean patients with type 2 diabetes mellitus: a 16-week, randomized, double-blind, placebo-controlled phase III trial. Diabetes Obes Metab. 2015; 17: 309-312. Ref.: https://goo.gl/no1fnN 
10. Pareek A, Chandurkar N, Thomas N, Viswanathan V, Deshpande A, et al. Efficacy and safety of hydroxychloroquine in the treatment of type 2 diabetes mellitus: a double blind, randomized comparison with pioglitazone. 2014; 30: 1257-1266. Ref.: https://goo.gl/MKEc1g

11. Quatraro A, Consoli G, Magno M, Caretta F, Nardozza A, et al. Hydroxychloroquine in decompensated, treatment-refractory noninsulin-dependent diabetes mellitus. a new job for an old drug? Ann Intern Med 1990; 112: 678-681. Ref.: https://goo.gl/1NE9ET

12. Baidya A, Chakravarti HN, Saraogi RK, Gupta A, Ahmed R, et al. Efficacy of Maximum and Optimum Doses of Hydroxychloroquine Added to Patients With Poorly Controlled Type 2 Diabetes on Stable Insulin Therapy along with Glimepiride And Metformin: Association of High-Sensitive C-Reactive Protein (Hs-CRP) and Glycosylated Haemoglobin (HbA1c). Endocrinol Metab Syndr. 2018; 7: 283. Ref.: https://goo.gl/UL29JC

13. Jagnani VK, Bhattacharya NR, Satpathy SC, Hasda GC, Chakraborty S. Effect of Hydroxychloroquine on Type 2 Diabetes Mellitus Unresponsive to More Than Two Oral Antidiabetic Agents. J Diabetes Metab. 2017; 8: 771. Ref.: https://goo.gl/e6LbQG

14. Baidya A, Kumar M, Pathak SK, Ahmed R. Study of comparative effect of hydroxychloroquine and vildagliptin on glycaemic efficacy and $\mathrm{HbA} 1 \mathrm{c}$ in type 2 diabetes patients who were inadequately controlled with metformin and glimepiride dual therapy; JMSCR. 2018; 6: 409-415. Ref.: https://goo.gl/qmhJhd

15. Sing SP, Sinha MK, Alok KA, Ranjan R. Comparative Study to Evaluate Effect of Hydroxychloroquine Versus Sitagliptin as Add on Therapy in Patients with Type 2 Diabetes Inadequately Controlled on Combination with Metformin and Gliclazide: A Multicenter, Observational Trial. Sch J App Med Sci. 2018; 6: 2150-2156

16. Meneilly GS, Elliott T, Tessier D, Hards L, Tildsesley H. NIDDM in the elderly. Diabetes Care. 1996; 19 : 1320-1325. Ref.: https://goo.gl/Zt5YQ8

17. Yang SH, Dou KF, Song WJ. Prevalence of diabetes among men and women in China. N Engl J Med. 2010; 362: 2425-2426. https://goo.gl/fbvMDf

18. Nathan DM, Buse JB, Davidson MB, Heine RJ, Holman RR, et al. Management of hyperglycaemia in type 2 diabetes: a consensus algorithm for the initiation and adjustment of therapy. A consensus statement from the American Diabetes Association and the European Association for the Study of Diabetes. Diabetologia. 2006; 49: 1711-1721. Ref.: https://goo.gl/SpbEMH

19. Hepburn DA, MacLeod KM, Pell AC, Scougal IJ, Frier BM. Frequency and symptoms of hypoglycaemia experienced by patients with type 2 diabetes treated with insulin. Diabet Med. 1993; 10: 231-237. Ref.: https://goo.gl/yuE9gT 